\title{
NEONATO DE MADRE CON PREECLAMPSIA: RIESGO PARA TODA LA VIDA
}

\author{
Carmen Rosa Dávila Aliaga ${ }^{1}$
}

\begin{abstract}
RESUMEN
Preeclampsia es una patología que afecta a las gestantes y una de las principales causas de muerte materna en el Perú y en el mundo, generando morbilidad tanto en la madre como en su recién nacido, dentro los problemas más frecuentes en la etapa neonatal que se asocian a ésta patología en los recién nacidos tenemos: restricción de crecimiento intrauterino, pequeño para edad gestacional, bajo peso, prematuridad, trastornos metabólicos, trastornos de coagulación, hemorragia intraventricular, hiperbilirrubinemia, dificultad respiratoria, persistencia de conducto arterioso, enterocolitis necrosante, hipotensión, retinopatía, gastritis erosiva, predisposición a cuadros infecciosos, como sepsis debido a déficit en la actividad de granulocitos, quimiotaxis y citoquinas, etc. La repercusión de esta enfermedad continúa durante toda la vida asociando riesgo de diabetes mellitus, hiperlipidemias, hipertensión arterial, coronariopatía, entre otras.
\end{abstract}

Palabras clave: Recién nacido; prematuro; restricción de crecimiento intrauterino; preeclampsia (Fuente: DeCS BIREME).

\section{NEWBORN'S MOTHER WITH PREECLAMPSIA: RISK FOR ALL OF THE LIFE}

\begin{abstract}
Preeclampsia is a condition that affects pregnant women and is one of the leading causes of maternal death in Peru and the world, causing morbidity in both the mother and her newborn, in the most common problems in the neonatal age that associated with this pathology in neonates are: intrauterine growth restriction, small for gestational age, low birth weight, prematurity, metabolic disorders, clotting disorders, intraventricular hemorrhage, hyperbilirubinemia, respiratory distress, patent ductus arteriosus, necrotizing enterocolitis, hypotension, retinopathy, erosive gastritis, predisposition to infectious processes, including sepsis due to deficit in the activity of granulocyte chemotaxis and cytokines, etc. The impact of this disease continues throughout the life associating risk of diabetes mellitus, hyperlipidemia, hypertension, coronary artery disease, among others.
\end{abstract}

Key words: Newborn; Premature; Intrauterine growth restriction; Pre-eclampsia (Source:MeSH NLM).

\section{INTRODUCCIÓN}

La Preeclampsia fue descrita desde la antigua Grecia y se han postulado diferentes hipótesis acerca de las causas que la provocan, sin embargo hasta la fecha no se ha establecido fehacientemente. Los trastornos hipertensivos del embarazo constituyen una de las causas de muerte materna, junto con la hemorragia y la infección ${ }^{1,2}$.

La incidencia está influida por la paridad, predisposición racial, genética y factores ambientales ${ }^{2-4}$. Afecta entre el $5-10 \%{ }^{5}$ (promedio $7 \%{ }^{6,7}$ ) de los embarazos.

La Hipertensión Inducida por el Embarazo se clasifica en: Hipertensión sola sin proteinuria o edema patológico, Preeclampsia (leve, severa) y Eclampsia ${ }^{8}$.

Preeclampsia es la hipertensión arterial inducida por el embarazo más proteinuria o edema generalizado, en una gestante previamente normotensa ${ }^{2,9}$. Eclampsia es la aparición de convulsiones tónico-clónicas generalizadas, no causadas por epilepsia u otro cuadro convulsivo en una paciente con hipertensión inducida por el embarazo.

HELLP es una complicación grave de los transtornos hipertensivos del embarazo y consiste en $(\mathrm{H})$ Hemólisis, (ÉL) Enzimas hepáticas elevadas, (LP) Recuento de plaquetas bajo o Trombocitopenia, y está asociado a morbimortalidad materna y fetal elevadas ${ }^{8,10,11}$.

En la actualidad se conoce que ésta enfermedad tiene seria repercusión en el recién nacido, elevando así su morbilidad e inclusive llegando a la mortalidad ${ }^{9}$.

\section{FISIOPATOLOGÍA}

Se ha encontrado asociación entre la patología placentaria (peso anormal, maduración vellosa acelerada, arteriopatía decidual y morbilidad neonatal severa ó muerte ${ }^{3,12}$.

\footnotetext{
Médico Pediatra Neonatóloga. Profesor de la Facultad de Medicina Humana de la Universidad Nacional Federico Villareal y de la Universidad Particular San Juan Bautista. Directora Ejecutiva de Docencia, Investigación y Atención en Neonatología en el Instituto Nacional Materno Perinatal.Lima-Perú.
} 
La preeclampsia reduce la perfusión placentaria, generando reducción del flujo sanguíneo úteroplacentario que lleva a su vez a hipoxia fetoplacentaria, el cual es un factor importante en la patogénesis de retardo de crecimiento intrauterino, asfixia y enterocolitis necrotizante (NEC), entre otras ${ }^{13-16}$.

Se ha reportado elevada concentración de endotelina (ET) en cordón umbilical, potente agente vasoconstrictor que se libera en el endotelio vascular y contribuye a la lesión miocárdica que se encuentra en algunos recién nacidos $^{5,16}$

Altuham y cols, midieron el nivel de paraoxonasa (PON)1 , molécula que permite determinar el nivel antioxidatnte (TAS), encontrando en hijos de madre preeclámptica aumento de los niveles de TAS en sangre del cordón umbilical, lo cual podría indicar que el feto responde contra el daño oxidativo causado por un aumento del estrés oxidativo en la madre. El aumento de la presión de oxígeno durante el parto causa elevación significativa de los niveles de radicales libres de oxígeno en recién nacidos $^{17-19}$. Además, los bebés prematuros están más expuestos a los efectos tóxicos de los radicales libres de oxígeno dado que se someten a intervenciones, como reanimación y ventilación mecánica ${ }^{20}$.

Se consideran factores angiogénicos (factor de crecimiento placentario, endogelina soluble y fms-like tirosin kinasa-1), como predictores de complicaciones maternas y neonatales, como prematuridad y retardo de crecimiento intrauterino ${ }^{1,21}$. Así como trombocitopenia neonatal en la sangre del cordón umbilical de los recién nacidos nacidos de madres con preeclampsia ${ }^{22}$.

Al parecer el desequilibrio entre los factores angiogénicos y antiangiogénicos se asocian con resultados adversos maternos y neonatales. Otro factor importante en la neovascularización es el factor de crecimiento endotelial vascular (VEGF) potente regulador del desarrollo vascular durante la embriogénesis, así como la formación de vasos sanguíneos y supervivencia en la edad adulta ${ }^{23-28}$.

La alteración de la angiogénesis en la preeclampsia se demuestra con la detección de altos niveles de varios factores antiangiogénicos, como receptor de VEGF 1 (VEGFR-1), endogelina y bajos niveles de factores angiogénicos tales VEGF en la preeclampsia ${ }^{29-33}$. La combinación de estos resultados también pueden explicar el aumento de la incidencia de DBP en recién nacidos prematuros nacidos de madres con preeclampsia en comparación con los nacidos de madres normotensos ${ }^{32}$.

Kalay reportó en los hijos de madre preeclámptica bajas concentraciones de factor de crecimiento endotelial vascular (VEGF) y altas de VEGFR-1 lo cual parece estar en relación con severidad de dificultad respiratoria, lo que sugiere que VEGF puede ser uno de los principales componentes de la maduración pulmonar ${ }^{33,34}$.
Kahamanan encontró en las madres preeclámpticas homocisteína elevada (20 -30\%) la cual actúa sobre las paredes de los vasos sanguíneos, generando disfunción endotelial, especialmente dentro de la placenta y vasculatura, esta elevación también estuvo presente en el recién nacido ${ }^{22,35-38}$.

Turumen encontró en hijos de madres con preeclámpsia severa mayor reacción inflamatoria sistémica, citoquinas pro inflamatorias, activación de fagocitos, elevada expresión de molécula de adhesión CD11b y proteína $C$ reactiva $(\mathrm{PCR})$ elevada $^{39-41}$.

La presencia del alelo APOE-e4 en la madre preeclámptica se asoció con cerca de ocho veces más riesgo de preeclampsia (OR ajustada $=8,4 ; \mathrm{IC}$ del 95\%: 2,51 a $28,17 ; p=0,001)$. Además mayores niveles de Trigliceridos y LDL-Colesterol y niveles más bajos de HDL-Colesterol se encontraron en las mujeres embarazadas con preeclampsia severa cuyos recién nacidos eran portadores del alelo APOE-e4. Del mismo modo se aprecian niveles mayores de lípidos en los recién nacidos, lo cual podría asociar a riesgo de dislipidemias en edad adulta ${ }^{42}$ (Tabla 1 ).

\begin{tabular}{ll}
\hline \multicolumn{1}{c}{ Tabla 1. Daño relacionado } & Daño relacionado \\
\hline Homocisteina & Disfunción endotelial \\
Fms-like tirosina quinasa (sFlt-1) & Plaquetopenia \\
\hline Endotelina & Daño miocardico \\
\hline Troponina & Daño miocardico \\
\hline Apolipoprotenia E & Trastorno lipídico \\
Factor de crecimiento enotelial & Alteración pulmonar \\
vascular (VEGF) & \\
25-Hidroxivitamina D & \\
\hline
\end{tabular}

En estudio experimental simulando hijos de madres preeclampticas se encontró un número significativamente mayor de células neuronales apoptóticas en

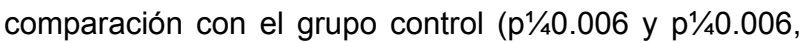
respectivamente). Fue también significativamente mayor el número de asfícticos, pequeños para la edad gestacional e hipoplasia cerebral. El pronóstico para el resultado neurológico es mucho peor cuando la asfixia perinatal se produce en los recién nacidos de madres con preeclampsia ${ }^{43}$.

\section{MORBILIDAD ASOCIADA EN LA ETAPA NEONATAL}

En el Hospital E. Rebagliati (Lima-Perú) en 1999 reportaron una mortalidad de $25 \%$ en aquellos hijos de madre preeclámptica de muy bajo peso al nacer (<1250gr), del mismo modo una elevada morbilidad como dificultad respiratoria $20,7 \%$, hemorragia intraventricular $16,9 \%$, hipoglicemia $16,9 \%$, sepsis $13,2 \%$ asfixia $9,4 \% ; 30 \%$ de esta población requirió reanimación y ventilación asistida ${ }^{44}$.

En el Instituto Nacional Materno Perinatal entre 1999-2000 reportaron en hijos de madres preeclámpticas, eclámptica 
y Síndrome HELLP, $50 \%$ eran pretérminos, hipoglicemia en $25,2 \%$, hiperglicemia $10,4 \%$, enfermedad de membrana hialina $8,9 \%$, hemorragia intrtaventricular $5,3 \%$, anemia $10,7 \%$, plaquetopenia $8,7 \%$ CID $5,9 \%$ y $16,9 \%$ de hijos de madre con preeclampsia severa fallecieron. Para el $2011,13 \%$ de las gestantes desarrollaron preeclampsia ó eclampsia. En el 2012 el 8,26\% de las gestantes desarrollaron algún trastorno hipertensivo del embarazo (preeclampsia 7,8\%, eclampsia 0,11\% y Sindrome HELLP $0,32 \%$ ). En el 2013 el 8,1\% de las gestantes desarrollaron algún trastorno hipertensivo del embarazo (preclampsia $7,65 \%$, eclampsia $0,12 \%$ y Sindrome HELLP $0,32 \%$ ). En el 2014 el 5,6\% de las gestantes desarrollaron algún trastorno hipertensivo del embarazo ( preeclampsia 5,3\%, eclampsia $0,1 \%$ y Sindrome HELLP 0, 2\%).

En el 2014, OMS publicó un trabajo realizado en 29 países del mundo, estableciendo una incidencia de preeclampsia de $2,16 \%$ y eclampsia $0,29 \%$, de un total de 313030 gestantes en su base de datos. Encontraron la asociación de preeclampsia y eclampsia como factor de riesgo de muerte fetal OR 3,12 (2,77-3,51), muerte neonatal temprana OR $2,71(2,28-3,21)$, prematuridad OR $3,02(2,73-3,34)$, ingresos a UCIN OR $3,45(3,21-3,75){ }^{45}$.

Dentro de los hallazgos encontrados en los hijos de madres preeclámpticas existe evidencia de: Retardo de crecimiento intrauterino, Pequeño para edad gestacional, prematuridad, ${ }^{47}$ trastornos metabólicos, como hipoglicemia ó hiperglicemia, hipocalcemia e hipopiridoxinemia;, trastornos de coagulación: plaquetopenia ${ }^{48,49}$ hipofibrinogenemia, tiempo de tromboplastina parcial prolongado. Además Hemorragia intraventricular, ${ }^{50}$ hiperbilirrubinemia, dificultad respiratoria (taquipnea transitoria, displasia broncopulmonar), persistencia de conducto arterioso, hipotensión, retinopatía, gastritis erosiva, predisposición a cuadros infecciosos, como sepsis debido a déficit en la actividad de granulocitos, quimiotaxis y citoquinas, población de células de defensa así como CD34+ disminuidas, se asocia neutropenia y esto a su vez predispone a infección por hongos en particular cándida ${ }^{21,51,52}$.

La enterocolitis necrotizante (NEC) es una importante causa de morbimortalidad en recién nacidos, Cetinkaya encontró mayor incidencia de NEC en recién nacidos de madres con preeclampsia $(22,9 \%)$ de inicio precoz y mayor duración y severidad, con OR de 1,74 (intervalo de confianza del $95 \%$ : 0,64 a 0,92$)^{53}$.

Se detectó disfunción diastólica del ventrículo izquierdo (DDVI) en los bebés prematuros nacidos de madres con preeclampsia en la primera semana después del parto. DDVI se asoció con una mayor incidencia de problemas respiratorios, taquipnea transitoria del recién nacido, mayor duración de la necesidad de oxígeno, y retraso del crecimiento intrauterino ${ }^{10,16}$.

La incidencia de DBP en los neonatos prematuros nacidos de madres preeclámpticas $(38,5 \%)$ fue significativamente mayor que en los nacidos de madres normotensas $(19,5 \%)^{38,54}$.

Cosar reportó riesgo de parálisis cerebral 1,5 veces mayor en los recién nacidos con este antecedente ${ }^{44,55}$. También hay reporte de leucomalacia periventricular quística ${ }^{12}$.

Abah y cols encontraron en los hijos de mujeres que experimentaron preeclampsia recurrente aumento en el riesgo de bajo peso al nacer y muy prematuros (OR = 3,77 , IC del $95 \%=2,77$ a 5,13 y OR $=3,66$, IC del $95 \%=$ 2,66-5,03, respectivamente) ${ }^{56}$ (Tabla 2).

Tabla 2. Morbilidad neonatal asociada a preeclampsia materna

\begin{tabular}{|l|}
\hline Retardo de crecimiento intrauterino \\
\hline Prematuridad \\
\hline Bajo peso \\
\hline Pequeño para edad gestacional (PEG) \\
\hline Trastornos metabólicos (Hipoglicemia, hipocalcemia) \\
\hline Plaquetopenia \\
\hline Neutropenia \\
\hline Policitemia \\
\hline Trastornos de coagulación \\
\hline Taquipnea Transitoria del recién nacido \\
\hline Hemorragia intraventricular \\
\hline Asfixia \\
\hline Hipoacusia neurosensorial \\
\hline Hipoplasia cerebral \\
\hline Sepsis \\
\hline Infecciones por hongos \\
\hline Retinopatía \\
\hline Ictericia \\
\hline Gastritis \\
\hline Enterocolitis necrotizante \\
\hline Hipotensión \\
\hline Daño miocárdico \\
\hline Persistencia de ductus arterioso \\
\hline Disfunción diastólica de ventrículo izquierdo \\
\hline Acidosis metabolica \\
\hline Insuficiencia renal \\
Hipotiroidismo \\
\hline Muerte neonatal \\
\hline
\end{tabular}

\section{MORBILIDAD EN LA ETAPA POSTNATAL}

En la etapa postneonatal en los hijos de madre con preeclampsia severa se reporta pobre ganancia ponderal, inapetencia, alteraciones de aprendizaje, hiperactividad, trastornos de comportamiento y trastornos del neurodesarrollo.

Probablemente dado que la preeclampsia generalmente ocurre en la gestación, a inicios del segundo trimestre, cuando justamente se están estableciendo los controles metabólicos en recién nacido, se establecen trastornos relacionados en etapa postneonatal. Cada vez es mayor la información de la repercusión del hijo de madre preeclámptica con respecto a su vida en la etapa adulta y en la que se evidencia trastornos de hipertensión arterial, 
ateroesclerosis, cardiopatía coronaria, dislipidemias, diabetes no insulina dependiente y obesidad $4-6,9,12,19,57-59$ (Tabla 3).

Tabla 3. Morbilidad postnatal asociada a preeclampsia materna

\begin{tabular}{l}
\hline Desórdenes del neurodesarrollo \\
Trastornos de aprendizaje \\
Ateroesclerosis \\
Diabetes mellitus \\
Coronariopatía \\
Hipertensión arterial \\
Infarto de miocardio \\
Obesidad \\
Trastorno tiroideo
\end{tabular}

\section{CONCLUSIONES}

La preeclampsia materna es una enfermedad materna con seria repercusión en el producto. Esta población de neonatos, presenta mayor tiempo de hospitalización y son responsables de elevación de cifras de mortalidad.

Los hijos de madre preeclámptica severa generalmente cursan con elevada morbilidad y por ende mortalidad con respecto a la población de niños del mismo peso y/o edad gestacional sin el antecedente de preeclampsia; generando mayor gasto de insumos, mayor tiempo de ocupación de cama en el área de cuidados intensivos y elevación de la morbimortalidad, con la debida implicancia negativa en los correspondientes indicadores de calidad. Es muy importante reconocer los factores de riesgo maternos, trabajar en la prevención de esta patología, realizar el diagnóstico y manejo oportuno de la gestante.

\section{REFERENCIAS BIBLIOGRÁFICAS}

1. Esplin MS, Fausett MB, Fraser A et al. Paternal and maternal Components of the Predisposition to Preeclampsia. N Engl J Med 2001; 344 (12): 867-72.

2. Myers JE, Baker PN. Hipertensive diseases and eclampsia. Curr Opin Obstet Gynecol 2002;14:119-125.

3. Skjerven R, Wilcox AJ, Lie RT. The Interval Between Pregnancies and the Risk of Preeclampsia. N Engl J Med 2002; 346 (1): 33-8

4. Roberts JM, pearson G, Cutler J, Lindheimer M. Summary of the NHLBI Working Group on Research on Hypertension During Pregnangy. Hypertension 2003; 41: 437-445

5. Anumba DOC, Robson SC. Management of pre-eclampsia and haemolysis, elevated liver enzymes, and low platelets syndrome. Curr Opin Obstet Gynecol 1999; 11(2): 146-56.

6. Tierney, McPhee, Papadakis. Diagnóstico clínico y tratamiento 2003. 38 a ed, México, Manual Moderno, 2003: 770-773

7. Masoura S, Kalogiannidis I, Makedou K, Theodoridis T, Koiou K, Gerou S, Athanasiadis A, Agorastos T. Biomarkers of endothelial dysfunction in preeclampsia and neonatal morbidity: a case-control study. European Journal of Obstetrics \& Gynecology and Reproductive Biology 175 (2014) 119-123

8. Vaticon D. Fisiología de la fecundación, embarazo, parto y lactancia, en: Tresguerres JAF. Fisiología Humana. México, Interamericana McGraw-Hill, 1992: 1086-1109.

9. Pridjian G, Puschett JB. Preeclampisa. Part 1: Clinical and Pathophysiologic Considerations. Obstet and Gynecol Survey 2002; 57 (9): 598-618

10. Estrada-Altamirano $A$, hernández-Pacheco JA, Cisneroscastolo M y García-Benitez CQ. Experiencia de la Unidad de Cuidados Intensivos Obstétricos del Instituto Nacional de Perinatología, 1993-1998. Perinatol Reprod Hum 2002; 16(2): 88-95

11. Dekker GA. Risk factors for Preeclampsia. Clin Obstet and Gynecol 1999; 42 (3): 422-35

12. Vinnars M, Nasiell J, Holmstrom G, Norman M,Westgren $\mathrm{M}$, and Papadogiannakis $\mathrm{N}$. Association between placental pathology and neonatal outcome in preeclampsia: a large cohort study. Hypertens Pregnancy, 2014; 33(2): 145-158

13. Redman CW. Current topic: pre-eclampsia and the placenta. Placenta. 1991;12:301-8.

14. Redman CW, Sargent IL. The pathogenesis of pre-eclampsia. Gynecol Obstet Fertil. 2001;29:518-22.

15. Roberts JM, Lain KY. Recent insights into the pathogenesis of pre-eclampsia. Placenta. 2002;23:359-72.

16. Cetinkaya M, Bostan O, Koksal N, Semizel E, Ozkan H and Cakır S. Early left ventricular diastolic dysfunction in premature infants born to preeclamptic mothers. J. Perinat. Med. 39 (2011) 89-95

17. Shoji H, Koletzko B. Oxidative stress and antioxidant protection in the perinatal period. Curr Opin Clin Nutr Metab Care 2007; 10: 324-328.

18. Thibeault DW. The precarious antioxidant defenses of the preterm infant. Am J Perinatol 2000; 17: 167-181.

19. Frank L. Development of antioxidant defenses in fetal life. Semin Neonatol 1998; 3: 173-182Xx

20. Altunhan $H$, Annagür $A$, Kurban $S$, Ertugrul $S$, Konak $M$ and Örs R. Total oxidant, antioxidant, and paraoxonase levels in babies born to pre-eclamptic mothers. J. Obstet. Gynaecol. Res. Vol. 39, No. 5: 898-904, May 2013

21. Sturtz WJ, Leef $\mathrm{KH}$, Mackley $A B$, et al. Homocy steine levels in preterm infants: is there an association with intraventricular hemorrhage? A prospective cohort study. BMC Pediatr 2007;7:38.

22. Kahramaner Z, Sutcuoglu S, Cicek E, Erdemir A, Cosar H, Turkoglu E, Colak A, and Ozer E. Homocysteine levels and its association with intraventricular hemorrhage in preterm infants born to preeclamptic mothers. J Matern Fetal Neonatal Med, 2013; 26(18): 1833-1837

23. Olsson AK, Dimberg A, Kreuger J, Welsch LC. VEGK receptor signaling in control of vascular function. Nat Rev Mol Cell Biol 2006;7:359-71.

24. Ribbatti D. The discovery of the placental growth factor and its role in angiogénesis: a historial review. Angiogenesis. 2008;11:215-21.

25. Leanos A, Campos L, Ramirez KL,Chinolla Z, Isordia I. Circulating angiogenic factors and urinary prolactin as predictors of adverse outcomes in women with preeclampsia. Hypertension 2013;61:1118-25.

26. Moor T, Crawford S, Solitro M, Frost S, Meyer B, Maynard S, Angiogenic factors for the prediction of preeclampsia in high rik women. Am J Obstet Gynecolol 2007;197:244-248

27. Guyton AC, Hall JE. Embarazo y lactancia en: Tratado de 
fisiología médica, $10^{a}$ ed, México, McGraw-Hill Interamericana 2001: 1135-45

28. Wilson MI, Goodwin TM, Pan VI, Ingles SA. Molecular epidemiology of preeclampsia. Obstet and Gynecol Survey 2003; 58(1):39-66

29. Levine RJ, Lam C, Qian C, Yu KF, Maynard SE, Sachs BP, Sibai BM, et al.; CPEP Study Group. Soluble endoglin and other circulating antiangiogenic factors in preeclampsia. $\mathrm{N}$ Engl J Med 2006;355: 992-1005.

30. Karumanchi SA, Lindheimer MD. Advances in the understanding of eclampsia. Curr Hypertens Rep 2008;10:305-312.

31. Foidart JM, Schaaps JP, Chantraine F, Munaut C, Lorquet S. Dysregulation of anti-angiogenic agents (sFlt-1, PLGF, and sEndoglin) in preeclampsia-a step forward but not the definitive answer. J Reprod Immunol 2009;82:106-111.

32. Ozkan H, Cetinkaya M \& Koksal N. Increased incidence of bronchopulmonary dysplasia in preterm infants exposed to preeclampsia. The Journal of Maternal-Fetal and Neonatal Medicine, 2012; 25(12): 2681-2685

33. Mutter WP, Karumanchi SA. Molecular mechanisms of preeclampsia. Microvasc Res 2008;75:1-8.

34. Kalay S, Cakcak B, Oztekin O, Tezel G, Tosun O, Akcakus $\mathrm{M}$, and Oygur $\mathrm{N}$. The role of VEGF and its soluble receptor VEGFR-1 in preterm newborns of preeclamptic mothers with RDS. J Matern Fetal Neonatal Med, 2013; 26(10): 978-983

35. Mignini LE, Latthe PM, Villar J, et al. Mapping the theories of preeclampsia: the role of homocysteine. Obstet Gynecol 2005;105: 411-25.

36. Cotter AM, Molloy JM, Scott JM, Daly SF. Elevated plasma homocysteine in early pregnancy: a risk factor for the development of severe preeclampsia. Am J Obstet Gynecol 2001; 185:781-85.

37. Vollset SE, Refsum H, Irgens LM, et al. Plasma total homocysteine, pregnancy complications, and adverse pregnancy outcomes: the Hordaland Homocysteine Study. Am J Clin Nutr 2000;71: 962-68.

38. Molloy A, Millis J, McPartlin J, et al. Maternal and fetal plasma homocysteine concentrations at birth: the influence of folate, vitamin B12, and the 5, 10-methylenetetrahydrofolate reductasKAJA 9

39. Turunen R, Andersson S, Laivuori H, Kajantie E, Siitonen S, Repo $\mathrm{H}$, Nupponen I. Increased Postnatal Inflammation in Mechanically Ventilated Preterm Infants Born to Mothers with Early-Onset Preeclampsia. Neonatology 2011;100:241-247

40. Paternoster DM, Fantinato $S$, Stella $A$, Nanhornguè $K N$, Milani M, Plebani M, Nicolini U, Girolami A: C-reactive protein in hypertensive disorders in pregnancy. Clin Appl Thromb Hemost 2006; 12:330-337.

41. Torrance HL, Krediet TG, Vreman HJ, Visser GH, van Bel $\mathrm{F}$ : Oxidative stress and proinflammatory cytokine levels are increased in premature neonates of preeclamptic mothers with HELLP syndrome. Neonatology 2008;94:138-142.

42. Scioscia M, Robillard P, Hall D, Rademacher L, Williams $P$, \& Rademacher T. Inositol phosphoglycan P-type in infants of preeclamptic mothers. The Journal of Maternal-Fetal and Neonatal Medicine, 2012; 25(2): 193-195

43. Cosar H, Ozer E, Topel H, Kahramaner Z, Turkoglu E, Erdemir A, Sutcuoglu S, Bagriyanik A, and Ozer E. Neuronal apoptosis in the neonates born to preeclamptic mothers. $J$ Matern Fetal Neonatal Med, 2013; 26(11): 1143-1146

44. Oliveros Donohue, Miguel; Chirinos Rivera, Jorge; Mayorga Ricaldi, Guido. Morbimortalidad del recién nacido de muy bajo peso y enfermedad hipertensiva del embarazo severa. Diagnóstico. Volumen 42 No 3. Mayo - Junio 2003.

45. Abalos E, Cuesta C, Carroli G, Qureshi Z, Widmer M, Vogel JP, Souza JP. Pre-eclampsia, eclampsia and adverse maternal and perinatal outcomes: a secondary analysis of the World Health Organization Multicountry Survey on Maternal and Newborn Health. World Health Organization 2014

46. XiongX,MayesD,DemianczukNet al. Impactofpregnancyinduced hypertension on fetal growth. Am J Obstet Gynecol 1999; 180: 207-213.

47. Ananth CV, Peedicayil A, Savitz DA. Effect of hypertensive diseases in pregnancy on birthweight, gestational duration, and small-for-gestational-age births. Epidemiology 1995; 6: 391-395. KAH 18.

48. Bayram F, Ozerkan K, Cengiz C, et al. Perinatal asphyxia is associated with the umbilical cord nucleated red blood cell count in pre-eclamptic pregnancies. J Obstet Gynaecol 2010;30: 383-6.

49. Infante-Rivarde C, Rivard G, Yotow W, Theoret Y. Perinatal reference intervals for plasma homocysteine and factors influencing its concentration. Clin Chem 2002;48:1100-2.

50. Schneider S, Freerksen N, Maul H, Roehrig S, Fischer B and Hoeft B. Risk groups and maternal-neonatal complications of preeclampsia - Current results from the national German Perinatal Quality Registry. J. Perinat. Med. 39 (2011) 257265

51. Schneider S, Huy C, Schutz J, Diehl K. Smoking cessation during pregnancy: a systematic literature review. Drug Alcohol Rev. 2010;29:81-90.

52. Cetinkaya M, Ozkan H, Koksal N. Maternal preeclampsia is associated with increased risk of necrotizing enterocolitis in preterm infants. Early Human Development 88 (2012) 893898

53. O'Shea J, Davis P and Doyle L. Maternal preeclampsia and risk of bronchopulmonary dysplasia in preterm infants. Pediatric Research (Volume 71, Number 2, February 2012)

54. Thorngren-Jerneck K, Herbst A. Perinatal factors associated with cerebral palsy in children born in Sweden. Obstet Gynecol 2006;108:1499-505.

55. Mbah A, Alio A, Marty P, Bruder K, Wilson R, Salihu H. Recurrent versus isolated pre-eclampsia and risk of fetoinfant morbidity outcomes: racial/ethnic disparity. European Journal of Obstetrics \& Gynecology and Reproductive Biology 156 (2011) 23-28

56. Burrows RF. Platelet disorders in pregnancy. Curr Opin Obstet and Gynecol 2001; 13: 115-9.

57. Thornton C, Makris A, Ogle F, Tooher J, Hennessy A. Role of proteinuria in defining pre-eclampsia: Clinical outcomes for women and babies. Clinical and Experimental Pharmacology and Physiology (2010) 37, 466-470.

58. Mbah A, Alio A, Marty P, Bruder K, Wilson R, Salihu H. Recurrent versus isolated pre-eclampsia and risk of fetoinfant morbidity outcomes: racial/ethnic disparity European Journal of Obstetrics \& Gynecology and Reproductive Biology 156 (2011) 23-28. 